\section{Disposable drapes and gowns}

Trycare supply an extensive range of PPE products to meet the individual requirements of every member of the practice team. This includes an extensive range of disposable drapes and gowns.

Trycare's disposable sterile surgical gowns and drapes are made of a fluid-repellent, three-layer laminate of breathable material to ensure the comfort of the wearer during the operative procedure. They combine comfort and performance, providing practices with the very best available protection and working conditions. Fully compliant with EN13795 and CE Marked 93/42/EEC, they are available in a range of sizes to meet every need.

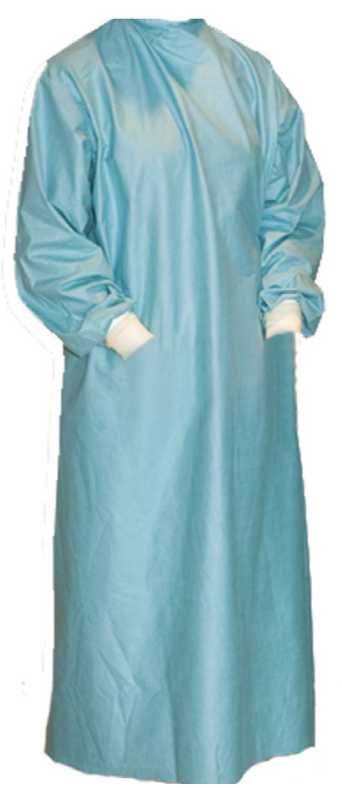

Trycare offer their drapes, gowns and ancillary items individually, in bulk packs and in a variety of offthe-shelf packs, designed to meet the requirements of different clinical procedures.

Should none of the above contain exactly what you want, then Trycare can supply customised drape kits designed to meet the individual needs of the practice.

For more information about Trycare's comprehensive PPE range, including drapes and gowns, contact your local Trycare Representative. Call 01274885 544 or visit www.trycare.co.uk.

\title{
A small amount of gold makes a huge difference
}

Dental scrap metal is possibly not at the top of your current practice agenda. What does the money that you occasionally receive on unwanted or broken crowns, bridges, PFMs, PFGs or other precious or semi-precious materials mean to you?

To some children it can mean the chance of a better life. There are more than 4.5 million children living in poverty in the UK.

Most dentists and dental professionals are unaware of the huge potential that the combined value of removed restorations represents, choosing to either ignore it, or retain it personally.

A new charity has been set up by two dentists who believe that patients, professionals and the public can be united in the belief that this money could be better used for the greater good - to help the many children's charities across the UK in their vital work.

Gold for Kids co-founder, Leticia Casanova said, 'my friend Kimberly MacGregor and I have always looked for ways in which we could do more to give back to our communities. As parents, the welfare of children always struck a chord with us - we were looking for a way that we could help many of the deserving causes out there. And that is how Gold for Kids was born
- a way for dental practices who are driven by their values to make a difference - collecting and turning restorations into cash for children's charities.

'We hope that many more people in the dental industry will become involved and in turn, we can raise more much-needed money to donate to crucial children's charities.'

To contribute to Gold for Kids:

1. Use the supplied, insured, pre-paid envelopes to send restorations (and jewellery) to Gold for Kids' secure storage facilities

2. Gold for Kids send the donations to a metal refinery to be processed. They recover the precious metals and turn them into bars, which are then sold to leading jewellery industry traders for cash

3. This is then donated to selected children's charities dedicated to improving the wellbeing and prospects of children in need. As a registered charity, Gold for Kids can reclaim gift aid on donations from UK taxpayers, increasing their value by $20 \%$.

To download a registration form and become a supporter of Gold for Kids, visit https://www. goldforkids.org/contact-us/.

\section{Twenty seconds to mix, place, dry and cure}

Exclusively available from Trycare,

Tokuyama Universal Bond is the only bonding agent that satisfies all three of The Dental Advisor's application requirements for a universal bonding agent. All the others only meet one or two.

Tokuyama Universal Bond is a twobottle, self-cured bonding system which delivers high adhesion values for all direct and indirect restorations. It can be used for direct anterior and posterior restorations with light-curing, dual-curing and selfcuring composite materials; intraoral repair of composite, porcelain fused to metal, metal and all-ceramic restorations without a primer; cementation of indirect restorations and veneers with light-curing, dual-curing and self-curing resin cements; bonding core build-ups; bonding denture resins to metal bases, clasps and attachments; repairing dentures; and bonding opaque resin to the metal bases of resin-faced stainless steel crowns.

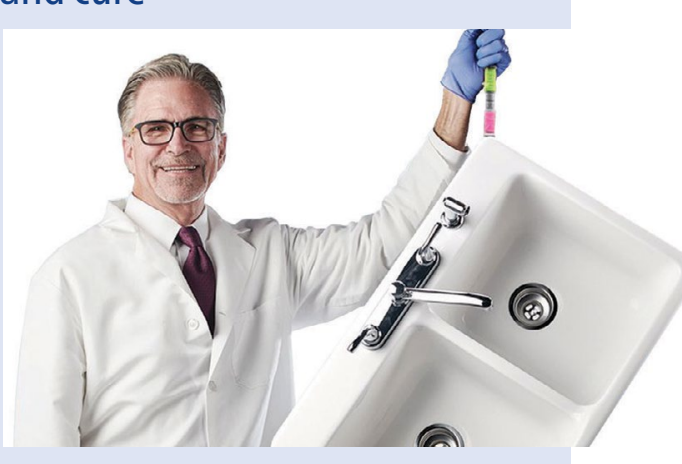

Compatible with all etching techniques, all forms of direct composite materials and indirect restorations (ceramic, zirconia or metal), with Tokuyama Universal Bond there is no need for light curing, no need to wait after application and no need for an activator or primer.

Tokuyama Universal Bond does not require any light curing step and takes just 20 seconds to mix, place, dry and cure!

For more information, contact your local Trycare Representative. Call 01274885544 or visit www.trycare.co.uk. 